PROCEEDINGS OF THE

AMERICAN MATHEMATICAL SOCIETY

Volume 125, Number 9, September 1997, Pages 2537-2541

S 0002-9939(97)04089-6

\title{
SMOOTH KUMMER SURFACES IN PROJECTIVE THREE-SPACE
}

\author{
THOMAS BAUER
}

(Communicated by Ron Donagi)

\begin{abstract}
In this note we prove the existence of smooth Kummer surfaces in projective three-space containing sixteen mutually disjoint smooth rational curves of any given degree.
\end{abstract}

\section{INTRODUCTION}

Let $X$ be a smooth quartic surface in projective three-space $\mathbb{P}^{3}$. As a consequence of Nikulin's theorem [6] $X$ is a Kummer surface if and only if it contains sixteen mutually disjoint smooth rational curves. The classical examples of smooth Kummer surfaces in $\mathbb{P}^{3}$ are due to Traynard (see [8] and [4]). They were rediscovered by Barth and Nieto [2] and independently by Naruki [5]. These quartic surfaces contain sixteen skew lines. In [1] it was shown by different methods that there also exist smooth quartic surfaces in $\mathbb{P}^{3}$ containing sixteen mutually disjoint smooth conics.

Motivated by these results it is then natural to ask if, for any given integer $d \geq 1$, there exist smooth quartic surfaces in $\mathbb{P}^{3}$ containing sixteen mutually disjoint smooth rational curves of degree $d$. The aim of this note is to show that the method of [1] can be generalized to answer this question in the affirmative. We show:

Theorem. For any integer $d \geq 1$ there is a three-dimensional family of smooth quartic surfaces in $\mathbb{P}^{3}$ containing sixteen mutually disjoint smooth rational curves of degree $d$.

We work throughout over the field $\mathbb{C}$ of complex numbers.

\section{Preliminaries}

Let $(A, L)$ be a polarized abelian surface of type $\left(1,2 d^{2}+1\right), d \geq 1$, and let $L$ be symmetric. Denote by $e_{1}, \ldots, e_{16}$ the halfperiods of $A$. We are going to consider the non-complete linear system

$$
\left|\mathcal{O}_{A}(2 L) \otimes \bigotimes_{i=1}^{16} \mathfrak{m}_{e_{i}}^{d}\right|^{ \pm}
$$

Received by the editors April 6, 1996.

1991 Mathematics Subject Classification. Primary 14J28; Secondary 14E25.

The author was supported by DFG contract Ba 423/7-1.

(C) 1997 American Mathematical Society 
of even respectively odd sections of $\mathcal{O}_{A}(2 L)$ vanishing in $e_{1}, \ldots, e_{16}$ to the order $d$. (As for the sign \pm we will always use the following convention: we take + if $d$ is even, and - if $d$ is odd.) A parameter count shows that the expected dimension of this linear system is 4 . In fact, we will show that it yields an embedding of the smooth Kummer surface $X$ of $A$ into $\mathbb{P}_{3}$ in the generic case. The linear system $(*)$ corresponds to a line bundle $M_{L}$ on $X$ such that

$$
\pi^{*} M_{L}=\mathcal{O}_{\widetilde{A}}\left(2 \sigma^{*} L-d \sum_{i=1}^{16} E_{i}\right)
$$

and

$$
H^{0}\left(X, M_{L}\right) \cong H^{0}\left(A, \mathcal{O}_{A}(2 L) \otimes \bigotimes_{i=1}^{16} \mathfrak{m}_{e_{i}}^{d}\right)^{ \pm}
$$

Here $\sigma: \widetilde{A} \longrightarrow A$ is the blow-up of $A$ in the halfperiods, $E_{1}, \ldots, E_{16} \subset \widetilde{A}$ are the exceptional curves and $\pi: \widetilde{A} \longrightarrow X$ is the canonical projection. The images of $E_{1}, \ldots, E_{16}$ under $\pi$ will be denoted by $D_{1}, \ldots, D_{16}$.

We will need the following lemma:

Lemma 1.1. Let the surfaces $A$ and $X$ and the line bundles $L$ and $M_{L}$ be as above. Further, let $C \subset X$ be an irreducible curve, different from $D_{1}, \ldots, D_{16}$, and let $F=\sigma_{*} \pi^{*} C$ be the corresponding symmetric curve on $A$. Then

(a) $M_{L}^{2}=4$ and $M_{L} D_{i}=d$ for $1 \leq i \leq 16$,

(b) $F^{2}=2 C^{2}+\sum_{i=1}^{16}$ mult $_{e_{i}}(F)^{2}$, and

(c) $L F=M_{L} C+\frac{d}{2} \sum_{i=1}^{16}$ mult $_{e_{i}}(F)$.

The proof consists in an obvious calculation.

\section{Bounding DEGREes AND MULTIPLICITIES}

Here we show two technical statements on the degrees and multiplicities of symmetric curves. We start with a lemma which bounds the degree of a symmetric curve on $A$ in terms of the degree of the corresponding curve on the smooth Kummer surface of $A$ :

Lemma 2.1. Let $C \subset X$ be an irreducible curve, different from $D_{1}, \ldots, D_{16}$, and let $F=\sigma_{*} \pi^{*} C$.

(a) If $M_{L} C=0$, then $L F \leq 2\left(1-C^{2}\right) d^{2}+16$.

(b) If $M_{L} C>0$, then $L F \leq 4\left(M_{L} C-\frac{C^{2}}{M_{L} C}\right) d^{2}+9 M_{L} C$.

Proof. For $\gamma \geq 0$ apply Hodge index to the line bundle $M_{L}$ and the divisor $C+\frac{\gamma}{d} D_{i}$ :

$$
M_{L}^{2}\left(C+\frac{\gamma}{d} D_{i}\right)^{2} \leq\left(M_{L} C+\frac{\gamma}{d} M_{L} D_{i}\right)^{2}
$$

Using Lemma 1.1(a) and the equality $C D_{i}=\operatorname{mult}_{e_{i}}(F)$ we get

$$
\text { mult }_{e_{i}}(F) \leq\left(\frac{\left(M_{L} C\right)^{2}}{8 \gamma}+\frac{\gamma}{8}+\frac{M_{L} C}{4}-\frac{C^{2}}{2 \gamma}\right) d+\frac{\gamma}{d} ;
$$


hence by Lemma 1.1(c)

$$
L F \leq\left(\frac{\left(M_{L} C\right)^{2}}{\gamma}+\gamma+2 M_{L} C-\frac{4 C^{2}}{\gamma}\right) d^{2}+M_{L} C+8 \gamma .
$$

Now the assertion follows by setting $\gamma=2$ in case $M_{L} C=0$ and by setting $\gamma=M_{L} C$ otherwise.

Further, we will need the following inequality on multiplicities of symmetric curves:

Lemma 2.2. Let $F \subset A$ be a symmetric curve such that $\mathcal{O}_{A}(F)$ is of type $(1, e)$ with e odd. Then

$$
\sum_{i=1}^{16} \text { mult }_{e_{i}}(F)^{2} \geq \frac{1}{16}\left(\sum_{i=1}^{16} \text { mult }_{e_{i}}(F)\right)^{2}+\frac{15}{4} .
$$

Proof. For $k \geq 0$ define the integers $n_{k}$ by

$$
n_{k}={ }_{\text {def }} \#\left\{i \mid m_{i}=k, 1 \leq i \leq 16\right\} .
$$

Abbreviating $m_{i}=\operatorname{mult}_{e_{i}}(F)$ we then have

$$
\sum_{i=1}^{16} m_{i}=\sum_{k \geq 0} k n_{k} \quad \text { and } \quad \sum_{i=1}^{16} m_{i}^{2}=\sum_{k \geq 0} k^{2} n_{k} .
$$

The polarized abelian surface $\left(A, \mathcal{O}_{A}(F)\right)$ is the pull-back of a principally polarized abelian surface $(B, P)$ via an isogeny $\varphi: A \longrightarrow B$ of odd degree. The Theta divisor $\Theta \in|P|$ passes through six halfperiods with multiplicity one and through ten halfperiods with even multiplicity. Therefore the symmetric divisor $F \in\left|\varphi^{*} P\right|$ is of odd multiplicity in six halfperiods and of even multiplicity in ten halfperiods or vice versa. So we have

$$
\sum_{k \equiv 0(2)} n_{k}=a \quad \text { and } \quad \sum_{k \equiv 1(2)} n_{k}=b,
$$

where $(a, b)=(6,10)$ or $(a, b)=(10,6)$.

Under the restriction (1) the difference

$$
\sum k^{2} n_{k}-\frac{1}{16}\left(\sum k n_{k}\right)^{2}
$$

is minimal, if for some integer $k_{0} \geq 0$ we have

$$
n_{k_{0}}=10, n_{k_{0}+1}=6 \text { or } n_{k_{0}}=6, n_{k_{0}+1}=10 .
$$

In this case we get $\sum k^{2} n_{k}-\frac{1}{16}\left(\sum k n_{k}\right)^{2}=\frac{15}{4}$, which implies the assertion of the lemma.

\section{Kummer surfaces With SiXteEn SKeW Rational CURVES OF GIVEN DEGREE}

The aim of this section is to show:

Theorem 3.1. Let $(A, L)$ be a polarized abelian surface of type $\left(1,2 d^{2}+1\right), d \geq 1$. Assume $\rho(A)=1$. Then the map $\varphi_{M_{L}}: X \longrightarrow \mathbb{P}^{3}$ defined by the linear system $\left|M_{L}\right|$ is an embedding. The image surface $\varphi_{M_{L}}(X)$ is a smooth quartic surface containing sixteen mutually disjoint smooth rational curves of degree $d$.

In particular, this implies the theorem stated in the introduction. 
Proof. Using Riemann-Roch, Kodaira vanishing and Lemma 1.1(a), we will be done as soon as we can show that $M_{L}$ is very ample. For $d=1$ this follows from [3], whereas for $d=2$ it follows from [1]. So we may assume $d \geq 3$ in the sequel.

(a) First we show that $M_{L}$ is globally generated. A possible base part $B$ of the system $\left|\mathcal{O}_{A}(2 L) \otimes \bigotimes_{i=1}^{16} \mathfrak{m}_{e_{i}}^{d}\right|^{ \pm}$is totally symmetric, so $B$ is algebraically equivalent to some even multiple of $L$, which is impossible for dimensional reasons. It remains the possibility that one - hence all - of the curves $D_{i}$ is fixed in $\left|M_{L}\right|$. So $M_{L}-$ $\mu \sum D_{i}$ is free for some $\mu \geq 1$. But $\left(M_{L}-\mu \sum D_{i}\right)^{2}=4-32 \mu d-32 \mu^{2}<0$, a contradiction.

(b) Our next claim is that $M_{L}$ is ample. Otherwise there is an irreducible (-2)curve $C \subset X$ such that $M_{L} C=0$. Lemma 1.1 shows that we have

$$
L F=\frac{d}{2} \sum m_{i} \quad \text { and } \quad F^{2}=-4+\sum m_{i}^{2}
$$

for the symmetric curve $F=\sigma_{*} \pi^{*} C$ with multiplicities $m_{i}=\operatorname{mult}_{e_{i}}(F)$. According to Lemma 2.1 the degree of $F$ is bounded by

$$
L F \leq 6 d^{2}+16 .
$$

Since $L$ is a primitive line bundle, the assumption on the Néron-Severi group of $A$ implies that $\mathcal{O}_{A}(F)$ is algebraically equivalent to some multiple $p L, p \geq 1$, thus we have $L F=p L^{2}=p\left(4 d^{2}+2\right)$, and then (2) implies $p=1$ because of our assumption $d \geq 3$. So we find

$$
8 d^{2}+4=2 L F=d \sum m_{i}
$$

and reduction mod $d$ shows that necessarily $d=4$. But in this case $\sum m_{i}$ would be odd, which is impossible (cf. [3]).

(c) Finally we prove that $M_{L}$ is very ample. Suppose the contrary. SaintDonat's criterion [7, Theorem 5.2 and Theorem 6.1(iii)] then implies the existence of an irreducible curve $C \subset X$ with $M_{L} C=2$ and $C^{2}=0$. So we have

$$
L F=2+\frac{d}{2} \sum m_{i} \quad \text { and } \quad F^{2}=\sum m_{i}^{2}
$$

for the corresponding symmetric curve $F=\sigma_{*} \pi^{*} C$. Lemma 2.1 yields the estimate

$$
L F \leq 8 d^{2}+18 \text {. }
$$

As above $\mathcal{O}_{A}(F)$ is algebraically equivalent to some multiple $p L, p \geq 1$, hence we get

$$
p\left(4 d^{2}+2\right)=p L^{2} \leq 8 d^{2}+18,
$$

which implies $p \leq 2$. If we had $p=2$ then reduction mod $d$ of the equation

$$
2\left(4 d^{2}+2\right)=2+\frac{d}{2} \sum m_{i}
$$

would give $d=4$. But in this case we have $\sum m_{i}=65$, which is impossible.

So the only remaining possibility is $p=1$, thus

$$
4 d^{2}+2=2+\frac{d}{2} \sum m_{i}=\sum m_{i}^{2} .
$$

But a numerical check shows that this contradicts Lemma 2.2. This completes the proof of the theorem. 
Remark 3.2. We conclude with a remark on the genericity assumption on the abelian surface $A$. It is certainly not true that the line bundle $M_{L}$ is very ample for every polarized abelian surface $(A, L)$ of type $\left(1,2 d^{2}+1\right)$. Consider for instance the case where $A=E_{1} \times E_{2}$ is a product of elliptic curves and $L=$ $\mathcal{O}_{A}\left(\{0\} \times E_{2}+\left(2 d^{2}+1\right) E_{1} \times\{0\}\right)$. Here, taking $C \subset X$ to be curve corresponding to $E_{1} \times\{0\}$, we have

$$
M_{L} C=1-2 d<0,
$$

so in this case $M_{L}$ is not even ample or globally generated.

\section{REFERENCES}

1. Barth, W., Bauer, Th.: Smooth quartic surfaces with 352 conics. Manuscripta Math. 85, 409417 (1994) MR 95j: 14045

2. Barth, W., Nieto, I.: Abelian surfaces of type $(1,3)$ and quartic surfaces with 16 skew lines. J. Algebraic Geometry 3, 173-222 (1994) MR 95e:14033

3. Bauer, Th.: Projective images of Kummer surfaces. Math. Ann. 299, 155-170 (1994) MR 95c: 14040

4. Godeaux, L.: Sur la surface du quatrième ordre contenant trente-deux droites. Académie Royale de Belgique, Bulletin de la Classe des Sciences, 5. sér., 25, 539-552 (1939) MR 4:253e

5. Naruki, I.: On smooth quartic embedding of Kummer surfaces. Proc. Japan Acad. 67, Ser. A, 223-225 (1991) MR 93b:14064

6. Nikulin, V.V.: On Kummer surfaces. Math. USSR Izvestija, Vol.9, No.2, 261-275 (1975)

7. Saint-Donat, B.: Projective models of K3 surfaces. Amer. J. Math. 96, 602-639 (1974) MR 51:518

8. Traynard, E.: Sur les fonctions thêta de deux variables et les surfaces hyperelliptiques. Ann. Scient. Éc. Norm. Sup., 3. sér., t. 24, 77-177 (1907)

Mathematisches Institut, Universität Erlangen-Nürnberg, Bismarckstrasse $1 \frac{1}{2}$, D91054 Erlangen, Germany

E-mail address: bauerth@mi.uni-erlangen.de

Current address: Department of Mathematics, University of California, Los Angeles, California 90024

E-mail address: tbauer@math.ucla.edu 